\title{
Refractory Ventricular Tachycardia in a Patient with a Left Ventricular Assist Device Successfully Treated with Stellate Ganglion Phototherapy: A Case Report
}

Tomoya Sato ${ }^{1}$, Rui Kamada ${ }^{1}$, Takuya Koizumi $^{2}$, Sakae Takenaka ${ }^{1}$, Shingo Tsujinaga ${ }^{1}$, Takao Konishi ${ }^{1}$, Takuma Sato ${ }^{1}$, Masaya Watanabe ${ }^{3}$, Toshiyuki Nagai ${ }^{1}$, and Toshihisa Anzai $^{1}$

${ }^{1}$ Hokkaido University Graduate School of Medicine

${ }^{2}$ Hokkaido University School of Medicine

${ }^{3}$ Hokkaido University

May 18, 2020

\begin{abstract}
Neuraxial modulation therapies, such as stellate ganglion block, thoracic epidural anesthesia, and cardiac sympathetic denervation, are effective for ventricular arrhythmias. However, these treatments are invasive and can increase the risk of bleeding and infection. In this case report, stellate ganglion phototherapy (SGP) was safely and effectively performed for refractory ventricular tachycardia in patients with a history of cardiac resynchronization therapy-defibrillator (CRT-D) and left ventricular assist device (LVAD) implantation for dilated cardiomyopathy. SGP might have the potential to treat refractory ventricular arrhythmias and can be useful because of its safety and simplicity.
\end{abstract}

\section{Hosted file}

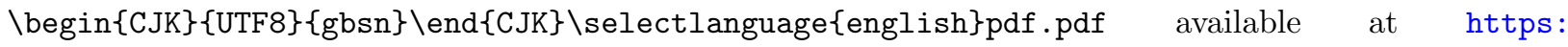
//authorea.com/users/323306/articles/452062-refractory-ventricular-tachycardia-ina-patient-with-a-left-ventricular-assist-device-successfully-treated-with-stellateganglion-phototherapy-a-case-report 